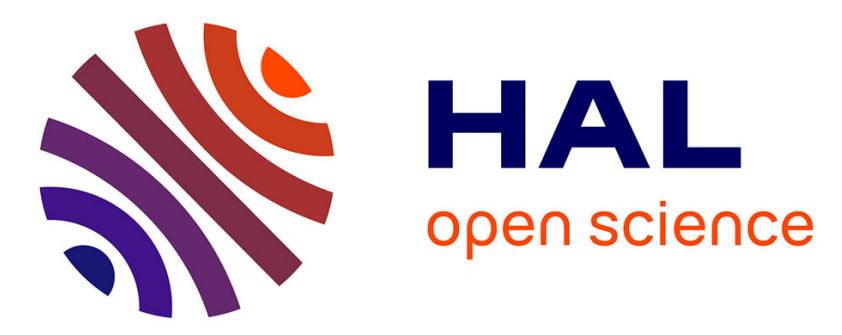

\title{
A Non-Hydrostatic Non-Dispersive Shallow Water Model
}

\author{
Didier Clamond, Denys Dutykh
}

\section{To cite this version:}

Didier Clamond, Denys Dutykh. A Non-Hydrostatic Non-Dispersive Shallow Water Model. Ph. Gourbesville et al. Advances in Hydroinformatics, Springer Singapore, pp.189-196, 2013, Springer Hydrogeology 2014, 978-981-4451-42-0. 10.1007/978-981-4451-42-0_16 . hal-00907110

\section{HAL Id: hal-00907110 https://hal.science/hal-00907110}

Submitted on 18 Dec 2018

HAL is a multi-disciplinary open access archive for the deposit and dissemination of scientific research documents, whether they are published or not. The documents may come from teaching and research institutions in France or abroad, or from public or private research centers.
L'archive ouverte pluridisciplinaire HAL, est destinée au dépôt et à la diffusion de documents scientifiques de niveau recherche, publiés ou non, émanant des établissements d'enseignement et de recherche français ou étrangers, des laboratoires publics ou privés.

\section{(1) $\$(0)$}

Distributed under a Creative Commons Attribution - NonCommercial - ShareAlikel 4.0 


\title{
A Non-Hydrostatic Non-Dispersive Shallow Water Model
}

\author{
Didier Clamond and Denys Dutykh
}

\begin{abstract}
An improvement of the nonlinear shallow water (or Saint-Venant) equations is proposed. The new model is designed to take into account the effects resulting from the large spatial and/or temporal variations of the seabed. The model is derived from a variational principle by choosing the appropriate shallow water ansatz and imposing suitable constraints. Thus, the derivation procedure does not explicitly involve any small parameter.
\end{abstract}

Keywords Varying bathymetry - Steep bottom • Modified Saint-Venant equations.

\section{Introduction}

The celebrated classical nonlinear shallow water (Saint-Venant) equations were derived for the first time in 1871 by Saint-Venant [1]. Currently, these equations are widely used in practice and the literature counts many thousands of publications devoted to the applications, validations, or numerical solutions of these equations.

Some important attempts have been also made to improve this model from physical point of view. The main attention was paid to various dispersive

D. Clamond $(\bowtie)$

Laboratoire J.-A. Dieudonné Parc Valrose, Université de Nice - Sophia Antipolis,

Nice 06108 Nice Cedex 2, France

e-mail: didier.clamond@gmail.com

D. Dutykh

Université de Savoie, Laboratoire de Mathématiques Appliquées Campus Scientifique,

LeBourget-du-Lac Cedex 73376, France

e-mail: denys.dutykh@univ-savoie.fr 
extensions of shallow water equations. The inclusion of dispersive effects resulted in a big family of the so-called Boussinesq-type equations (c.f., e.g., [2, 3]).

However, there are a few studies which attempt to include the bottom curvature effect into the classical Saint-Venant. One of the first studies in this direction is perhaps due to Dressler [4]. Much later, this research was pursued almost in the same time by Keller [5] and by Bouchut et al. [6]. We note that all these authors used some variant of the asymptotic expansion method. The present study is a further attempt to improve the classical Saint-Venant equations by including a better representation of the bottom shape. As a general derivation procedure, we choose a variational approach based on a relaxed Lagrangian principle [7].

In the next section, we present the derivation and discussion of some properties of the improved Saint-Venant equations. Then, we detail the hyperbolic structure in Sect. 3 and give a numerical example in Sect. 4. Finally, we underline some main conclusions of this study in Sect. 5 .

\section{Modified Saint-Venant Equations}

Consider an ideal incompressible fluid of constant density $\rho$. The horizontal independent variables are denoted by $\boldsymbol{x}=\left(x_{1}, x_{2}\right)$ and the upward vertical one by $y$. The origin of the Cartesian coordinate system is chosen such that the surface $y=0$ corresponds to the still water level. The fluid is bounded below by an impermeable bottom at $y=-d(\boldsymbol{x}, t)$ and above by an impermeable free surface at $y=\eta(\boldsymbol{x}, t)$. Usually, we assume that the total depth $h(\boldsymbol{x}, t)=d(\boldsymbol{x}, t)+\eta(\boldsymbol{x}, t)$ remains positive $h(\boldsymbol{x}, t) \geq h_{0}>0$ for all times $t$. Traditionally, in water wave modeling, the assumption of flow irrotationality is also adopted. The assumptions of fluid incompressibility and flow irrotationality lead to the Laplace equation for a velocity potential $\phi(\boldsymbol{x}, y, t)$.

It is well known that the water wave problem possesses several variational structures [8-10]. Recently, we proposed a relaxed Lagrangian variational principle which allows much more freedom for constructing approximations in comparison with classical formulations, namely the water wave equations can be derived as the critical point of the functional $\iiint \mathscr{L} d^{2} x d t$ involving the Lagrangian density [7]:

$$
\begin{aligned}
\mathscr{L}= & \left(\eta_{t}+\tilde{\boldsymbol{\mu}} \cdot \nabla \eta-\tilde{v}\right) \tilde{\phi}+\left(d_{t}+\check{\boldsymbol{\mu}} \cdot \nabla d+\check{v}\right) \check{\phi}-\frac{1}{2} g \eta^{2} \\
& +\int_{-d}^{\eta}\left[\boldsymbol{\mu} \cdot \boldsymbol{u}-\frac{1}{2} \boldsymbol{u}^{2}+v v-\frac{1}{2} v^{2}+\left(\nabla \cdot \boldsymbol{\mu}+v_{y}\right) \phi\right] d y,
\end{aligned}
$$

where $g$ is the acceleration due to gravity force, and $\nabla=\left(\partial_{x_{1}}, \partial_{x_{2}}\right)$ denotes the gradient operator in horizontal Cartesian coordinates. Other variables $\{\boldsymbol{u}, v, \boldsymbol{\mu}, v\}$ are the horizontal velocity, the vertical velocity, and the associated Lagrange multipliers, respectively. The last two additional variables $\{\boldsymbol{\mu}, v\}$ are called pseudo-velocities. They formally arise as Lagrange multipliers associated with the 
constraints $\boldsymbol{u}=\nabla \phi, v=\phi_{y}$. However, once these variables are introduced, the ansatz can be chosen regardless to their initial definition. The over 'tildes' and 'wedges' denote, respectively, a quantity traces computed at the free surface $y=$ $\eta(\boldsymbol{x}, t)$ and at the bottom $y=-d(\boldsymbol{x}, t)$. We shall also denote below with 'bars' the quantities averaged over the water depth. Note that the efficiency of the relaxed variational principle (1) relies on the extra freedom for constructing approximations.

In order to simplify the full water wave problem, we choose some approximate, but physically relevant, representations of all variables. In this study, we consider very long waves in shallow water. This means that the flow is mainly columnar [11] and that the dispersive effects are negligible. In other words, a vertical slice of the fluid moves like a rigid body. Thus, we choose a simple shallow water ansatz, which is independent of the vertical coordinate $y$, and such that the vertical velocity $v$ equals the one of the bottom, i.e.,

$$
\phi \approx \bar{\phi}(\boldsymbol{x}, t), \quad \boldsymbol{u}=\boldsymbol{\mu} \approx \overline{\boldsymbol{u}}(\boldsymbol{x}, t), \quad v=v \approx \check{v}(\boldsymbol{x}, t),
$$

where $\check{v}(\boldsymbol{x}, t)$ is the vertical velocity at the bottom. In the above ansatz, we take for simplicity the pseudo-velocity to be equal to the velocity field $\boldsymbol{\mu}=\boldsymbol{u}, v=v$. Note that in other situations, they can differ (see [7] for more examples). With this ansatz, the Lagrangian density (1) becomes

$$
\mathscr{L}=\left(h_{t}+\overline{\boldsymbol{u}} \cdot \nabla h+h \nabla \cdot \overline{\boldsymbol{u}}\right) \bar{\phi}-\frac{1}{2} g \eta^{2}+\frac{1}{2} h\left(\overline{\boldsymbol{u}}^{2}+\check{v}^{2}\right),
$$

where we introduced the total water depth $h=\eta+d$.

Now, we are going to impose one constraint by choosing a particular representation of the fluid vertical velocity $\check{v}(\boldsymbol{x}, t)$ at the bottom, namely we require fluid particles to follow the bottom profile:

$$
\check{v}=-d_{t}-\overline{\boldsymbol{u}} \cdot \nabla d .
$$

This last identity is nothing else but the bottom impermeability condition within ansatz (2). Substituting the relation (4) into Lagrangian density (3), the EulerLagrange equations yield

$$
\begin{gathered}
\delta \bar{\phi}: \quad 0=h_{t}+\nabla \cdot[h \overline{\boldsymbol{u}}], \\
\delta \overline{\boldsymbol{u}}: \quad \boldsymbol{0}=\overline{\boldsymbol{u}}-\nabla \bar{\phi}-\check{v} \nabla d, \\
\delta \eta: \quad 0=\bar{\phi}_{t}+g \eta+\overline{\boldsymbol{u}} \cdot \nabla \bar{\phi}-\frac{1}{2}\left(\overline{\boldsymbol{u}}^{2}+\check{v}^{2}\right) .
\end{gathered}
$$

Taking the gradient of (7) and eliminating $\bar{\phi}$ from (6) gives us this system of governing equations

$$
h_{t}+\nabla \cdot[h \overline{\boldsymbol{u}}]=0,
$$




$$
\partial_{t}[\overline{\boldsymbol{u}}-\check{v} \nabla d]+\nabla\left[g \eta+\frac{1}{2} \overline{\boldsymbol{u}}^{2}+\frac{1}{2} \check{\boldsymbol{v}}^{2}+\check{v} d_{t}\right]=0,
$$

together with the relations

$$
\overline{\boldsymbol{u}}=\nabla \bar{\phi}+\check{v} \nabla d, \quad \check{v}=-d_{t}-\overline{\boldsymbol{u}} \cdot \nabla d=-\frac{d_{t}+\nabla \bar{\phi} \cdot \nabla d}{1+|\nabla d|^{2}} .
$$

Further details on these equations and their variants can be found in [12].

\section{Hyperbolic Structure}

From now on, we consider Eqs. $(8,9)$ posed in 1D space for simplicity:

$$
\begin{gathered}
\partial_{t} h+\partial_{x}[h \bar{u}]=0, \\
\partial_{t}\left[\bar{u}-\check{v} \partial_{x} d\right]+\partial_{x}\left[g \eta+\frac{1}{2} \bar{u}^{2}+\frac{1}{2} \check{v}^{2}+\check{v} \partial_{t} d\right]=0 .
\end{gathered}
$$

In order to make appear conservative variables, we will introduce the potential velocity variable $U=\partial_{x} \bar{\phi}$. From Eq. (6) it is straightforward to see that $U$ satisfies the relation $U=\bar{u}-\check{v} \partial_{x} d$. Depth averaged and vertical bottom velocities can be also easily expressed in terms of the potential $U$. Consequently, using this new variable Eqs. $(10,11)$ can be rewritten as a system of conservation laws

$$
\partial_{t} w+\partial_{x} f(w)=0
$$

where the vector of conservative variables $w$ and the advective flux $f(w)$ are

$$
w=\left(\begin{array}{c}
h \\
U
\end{array}\right), \quad f(w)=\left(\begin{array}{c}
h \frac{U-\left(\partial_{t} d\right)\left(\partial_{x} d\right)}{1+\left(\partial_{x} d\right)^{2}} \\
g(h-d)+\frac{U^{2}-2 U\left(\partial_{x} d\right)\left(\partial_{t} d\right)-\left(\partial_{t} d\right)^{2}}{2\left[1+\left(\partial_{x} d\right)^{2}\right]}
\end{array}\right) .
$$

The Jacobian matrix of the advective flux $f(w)$ can be easily computed:

$$
\mathbb{A}(w)=\frac{\partial f(w)}{\partial w}=\frac{1}{1+\left(\partial_{x} d\right)^{2}}\left[\begin{array}{cc}
U-\left(\partial_{t} d\right)\left(\partial_{x} d\right) & h \\
g\left(1+\left(\partial_{x} d\right)^{2}\right) & U-\left(\partial_{t} d\right)\left(\partial_{x} d\right)
\end{array}\right] .
$$

The matrix $\mathbb{A}(w)$ has two distinct eigenvalues:

$$
\lambda^{ \pm}=\frac{U-\left(\partial_{t} d\right)\left(\partial_{x} d\right)}{1+\left(\partial_{x} d\right)^{2}} \pm c=\bar{u} \pm c, \quad c^{2} \equiv \frac{g h}{1+\left(\partial_{x} d\right)^{2}} .
$$


Physically, the quantity $c$ represents the phase celerity of long gravity waves. In the framework of the Saint-Venant equations, it is well known that $c=\sqrt{g h}$. Both expressions differ by the factor $1 / \sqrt{1+\left(\partial_{x} d\right)^{2}}$. In our model, the long waves are slowed down by strong bathymetric variations since fluid particles are constrained to follow the seabed.

\section{Numerical Experiment}

The equations are solved numerically with a spacial finite volume scheme together with a high-order adaptive time stepping. In order to obtain a higher-order scheme in space, we use a piecewise polynomial representation. This is achieved by various so-called reconstruction procedures, such as MUSCL TVD [13-15], UNO [16], ENO [17], WENO [18], and many others. In order to solve numerically the last system of equations, we apply a third-order Runge-Kutta scheme with four stages, with an embedded second-order method which is used to estimate the local error and, thus, to adapt the time step size. The model details and its performance can be found in [19]. Here, we focus of an illustrative example of the new modified Saint-Venant model: Wave generation by a sudden bottom uplift. This simple situation has some important implications to tsunami genesis problems.

The bottom is given by the following function:

$$
d(x, t)=d_{0}-a T(t) H\left(b^{2}-x^{2}\right)\left[\left(\frac{x}{b}\right)^{2}-1\right]^{2}, \quad T(t)=1-e^{-\alpha t},
$$

where $H(x)$ is the Heaviside step function, $a$ is the deformation amplitude, and $b$ is the half-length of the uplifting sea floor area. The function $T(t)$ provides a complete information on the dynamics of the bottom motion. In tsunami wave literature, it is called a dynamic scenario. Initially, the free surface is undisturbed and the velocity field is taken to be identically zero. The values of various parameter are given in Table 1, where one should note that the quantities are normalized such that the water depth and the acceleration due to gravity are equal to one.

Table 1 Values of various parameters used for the wave generation by a moving bottom

\begin{tabular}{ll}
\hline Slow uplift parameter $\alpha_{1}:$ & $2.0 \mathrm{~s}^{-1}$ \\
Fast uplift parameter $\alpha_{2}:$ & $12.0 \mathrm{~s}^{-1}$ \\
Gravity acceleration $g:$ & $1 \mathrm{~m} \mathrm{~s}^{-2}$ \\
Final simulation time $T:$ & $5 \mathrm{~s}$ \\
Undisturbed water depth $d_{0}:$ & $1 \mathrm{~m}$ \\
Deformation amplitude $a:$ & $0.25 \mathrm{~m}$ \\
Half-length of the uplift area $b:$ & $2.5 \mathrm{~m}$
\end{tabular}



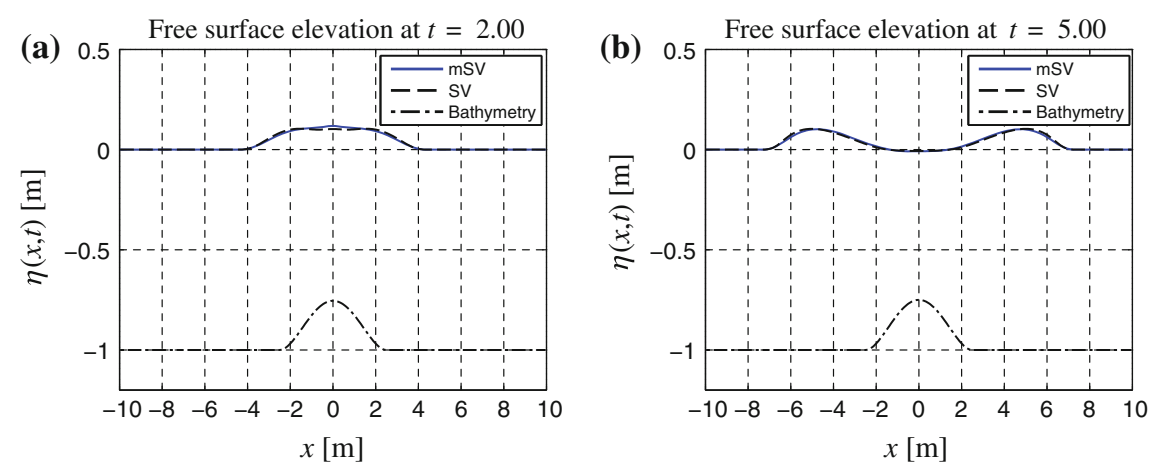

Fig. 1 Slow bottom uplift test case $\left(\alpha_{1}=2\right)$. a $t=2.0 \mathrm{~s}$. $\mathbf{b} t=5.0 \mathrm{~s}$

The numerical results of the moving bottom test case are shown on the figures below. On all these figures, the blue solid line corresponds to the modified SaintVenant equations (mSV), while the black dashed line refers to its classical counterpart (SV). The dash-dotted line shows the bottom profile which evolves in time as well.

First, we present numerical results (see Fig. 1) corresponding to a relatively slow uplift of a portion of the bottom $\left(\alpha_{1}=2.0 \mathrm{~s}^{-1}\right)$. There is a very good agreement between the two computations. We note that the amplitude of the bottom deformation $a / d=0.25$ is big which explains some small discrepancies on Fig. 1a between two models. This effect is rather due to the bottom shape than to its dynamic motion.

Second, we test the same situation but the bottom uplift is fast with the inverse characteristic time $\alpha_{2}=12.0 \mathrm{~s}^{-1}$. In this case, the differences between two models are very flagrant. As it can be seen on Fig. 2 that the modified Saint-Venant equations give a wave with almost two times higher amplitude. Some differences
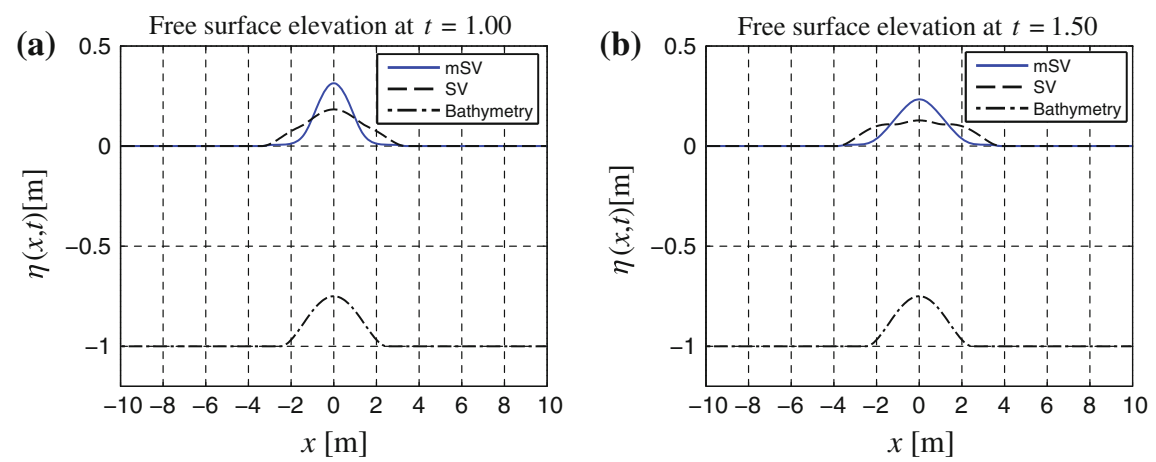

Fig. 2 Fast bottom uplift test case $\left(\alpha_{2}=12 \mathrm{~s}^{-1}\right)$. a $t=1.0 \mathrm{~s}$. b $t=1.5 \mathrm{~s}$ 

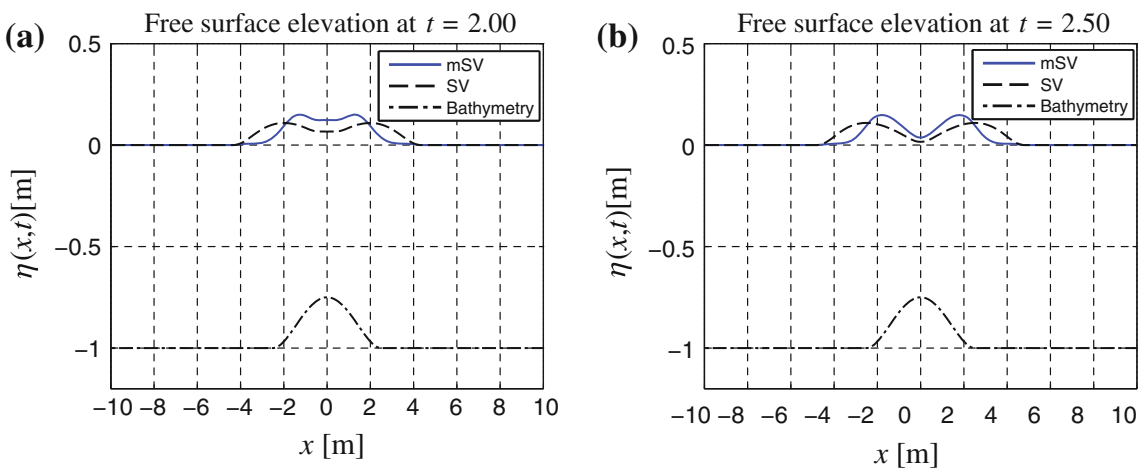

Fig. 3 Fast bottom uplift test case $\left(\alpha_{2}=12 \mathrm{~s}^{-1}\right)$. a $t=2.0 \mathrm{~s}$. b $t=2.5 \mathrm{~s}$

in the wave shape persist even during the propagation (see Fig. 3). This test case clearly shows another advantage of the modified Saint-Venant equations in better representation of the vertical velocity field.

\section{Conclusion}

We derived a non-hydrostatic non-dispersive model of shallow water type which takes into account large bathymetric variations. Previously, some attempt was already made in the literature to derive shallow water systems for arbitrary slopes and curvature [4-6]. However, our study contains a certain number of new elements with respect to the existing state of the art, namely our derivation procedure relies on a generalized Lagrangian principle of the water wave problem [7]. Moreover, we do not introduce any small parameter, and our approximation is made through the choice of a suitable constrained ansatz. Resulting governing equations have a simple form and physically sound structure. Another new element is the introduction of arbitrary bottom time variations.

The proposed model is discretized with a finite volume scheme with adaptive time stepping to capture the underlying complex dynamics. The performance of this scheme is then illustrated on several test cases. Some implications to tsunami wave modeling are also suggested at the end of this study.

Among various perspectives, we would like to underline the importance of a robust run-up algorithm development using the current model. This research should shift forward the accuracy and our comprehension of a water wave runup onto complex shores [20, 21]. 


\section{References}

1. de Saint-Venant, A. J. C. (1871). Théorie du mouvement non-permanent des eaux, avec application aux crues des rivières et à l'introduction des marées dans leur lit. C. R. Academic Science Paris, 73, 147-154.

2. Peregrine, D. H. (1967). Long waves on a beach. Journal of Fluid Mechanics, 27, 815-827.

3. Serre, F. (1953). Contribution à l'étude des écoulements permanents et variables dans les canaux. La Houille blanche, 8, 374-872.

4. Dressler, R. F. (1978). New nonlinear shallow-flow equations with curvature. Journal of Hydraulic Research, 16(3), 205-222.

5. Keller, J. B. (2003). Shallow-water theory for arbitrary slopes of the bottom. Journal of Fluid Mechanics, 489, 345-348.

6. Bouchut, F., Mangeney-Castelnau, A., Perthame, B., \& Vilotte, J.-P. (2003). A new model of Saint-Venant and Savage-Hutter type for gravity driven shallow water flows. C. R. Academic Science Paris, I(336), 531-536.

7. Clamond, D., \& Dutykh, D. (2012). Practical use of variational principles for modeling water waves. Physica D: Nonlinear Phenomena, 241(1), 25-36.

8. Petrov, A. A. (1964). Variational statement of the problem of liquid motion in a container of finite dimensions. Prikladnaia Mathematics Mekhanika, 28(4), 917-922.

9. Luke, J. C. (1967). A variational principle for a fluid with a free surface. Journal of Fluid Mechanics, 27, 375-397.

10. Zakharov, V. E. (1968). Stability of periodic waves of finite amplitude on the surface of a deep fluid. Journal of Applied Mechanical Technology Physics, 9, 190-194.

11. Miles, J. W., \& Salmon, R. (1985). Weakly dispersive nonlinear gravity waves. Journal of Fluid Mechanics, 157, 519-531.

12. Dutykh, D., \& Clamond, D. (2011). Shallow water equations for large bathymetry variations. Journal of Physics A: Mathematical Theory, 44, 332001.

13. Kolgan, N. E. (1975). Finite-difference schemes for computation of three dimensional solutions of gas dynamics and calculation of a flow over a body under an angle of attack. Uchenye Zapiski TsaGI [Science Notes Central Institute Aerodyn], 6(2), 1-6.

14. van Leer, B. (1979). Towards the ultimate conservative difference scheme V: a second order sequel to Godunov' method. Journal of Computer Physics, 32, 101-136.

15. van Leer, B. (2006). Upwind and high-resolution methods for compressible flow: From donor cell to residual-distribution schemes. Communications in Computational Physics, 1, 192-206.

16. Harten, A., \& Osher, S. (1987). Uniformly high-order accurate nonoscillatory schemes. $I$. SIAM Journal of Numerical Analytical, 24, 279-309.

17. Harten, A. (1989). ENO schemes with subcell resolution. Journal of Computer Physics, 83, $148-184$.

18. Xing, Y., \& Shu, C.-W. (2005). High order finite difference WENO schemes with the exact conservation property for the shallow water equations. Journal of Computer Physics, 208, 206-227.

19. Dutykh, D., \& Clamond, D. (2012). Modified shallow water equations for significantly varying bottoms. Submitted.

20. Dutykh, D., Katsaounis, T., \& Mitsotakis, D. (2011). Dispersive wave runup on non-uniform shores. In J. Fort, et al. (Eds.), Finite Volumes for Complex Applications VI - Problems \& Perspectives (pp. 389-397). pp Prague: Springer Berlin Heidelberg.

21. Dutykh, D., Labart, C., \& Mitsotakis, D. (2011). Long wave run-up on random beaches. Physics Review Letter, 107, 184504. 